\section{Prevalence and clinical outcomes of dilated cardiomyopathy (DCM) in dogs under first opinion veterinary care in the UK}

\section{Sarah Gane, Dan O'Neill, Adrian Boswood, David Church, David Brodbelt, Maddy Mattin}

RVC, Hertfordshire, UK

\section{OBJECTIVES}

This study aimed to identify the prevalence, management and outcomes of dilated cardiomyopathy (DCM) in first opinion UK practice.

\section{METHODS}

The study was a retrospective VetCompass study analysing a pooled database of first opinion veterinary clinical data. Inclusion criteria for DCM were any dog with a recorded diagnosis of either DCM or an unspecified cardiomyopathy. The study included all DCM cases recorded between 31/06/12 - 31/12/13. Signalment (breed, age, and sex), management and outcomes were extracted and described

\section{RESULTS}

There were 244 DCM cases identified from a study population of 455,557 dogs, giving an overall prevalence of $0.05 \%(95 \% \mathrm{Cl}: 0.05-0.06)$ with $157(64 \%)$ of the cases being male. The most common breeds were Labrador Retriever (11\%), Doberman Pincher (9\%) and Boxer ( $8 \%$ ). A murmur was not reported in $49 \%$ of cases and echocardiography was the most commonly used diagnostic test (48\%). Pimobendan (76\%) and furosemide $(62 \%)$ were the most commonly used treatments. Overall, 168 DCM cases died during the study period with 140 (83\%) involving euthanasia. DCM was found to be the primary cause of death in $123(73 \%)$ of cases that died.

\section{STATEMENT}

These findings on prevalence and severity can help to understand the impact of DCM at a population level to assist welfare prioritisation. The clinical management results can assist practitioners with benchmarking and clinical audit. 